Pacific

Journal of

Mathematics

\title{
ON CONSTRAINED EXTREMA
}

\author{
THOMAS VogeL
}

Volume $176 \quad$ No. 2

December 1996 


\title{
ON CONSTRAINED EXTREMA
}

\author{
THOMAS I. VOGEL
}

\section{Assume that $I$ and $J$ are smooth functionals defined on} a Hilbert space $H$. We derive sufficient conditions for $I$ to have a local minimum at $y$ subject to the constraint that $J$ is constantly $J(y)$.

The first order necessary condition for $I$ to have a constrained minimum at $y$ is that for some constant $\lambda, I_{y}^{\prime}+\lambda J_{y}^{\prime}$ is identically zero. Here $I_{y}^{\prime}$ and $J_{y}^{\prime}$ are the Fréchet derivatives of $I$ and $J$ at $y$. For the rest of the paper, we assume that $y$ in $H$ satisfies this necessary condition.

A common misapprehension (upon which much of the stability results for capillary surfaces has been based) is to assume that if the quadratic form $I_{y}^{\prime \prime}+\lambda J_{y}^{\prime \prime}$ is positive definite on the kernel of $J_{y}^{\prime}$ then $I$ has a local constrained minimum at $y$. This is not correct in a Hilbert space of infinite dimension; Finn [1] has supplied a counterexample in the unconstrained case, and the same difficulty will occur in the constrained case. In the unconstrained case, if (as often occurs in practice) the spectrum of $I_{y}^{\prime \prime}$ is discrete and 0 is not a cluster point of the spectrum, then $I_{y}^{\prime \prime}$ positive definite at a critical point $y$ implies that $I_{y}^{\prime \prime}$ is strongly positive, (i.e., there exists $k>0$ such that $I_{y}^{\prime \prime}(x) \geq k\|x\|^{2}$ holds for all $x$ ), and this in turn does imply that $y$ is a local minimum (see [2]). However, in the constrained case, things are not so easy. Even if $I_{y}^{\prime \prime}+\lambda J_{y}^{\prime \prime}$ has a nice spectrum (in some sense), it is not clear that $I_{y}^{\prime \prime}+\lambda J_{y}^{\prime \prime}$ being positive definite on the kernel of $J_{y}^{\prime}$ implies that this quadratic form is strongly positive on the kernel, nor that strong positivity implies that $y$ is a local minimum.

In [3], Maddocks obtained sufficient conditions for $I_{y}^{\prime \prime}+\lambda J_{y}^{\prime \prime}$ to be positive definite on the kernel of $J_{y}^{\prime}$. As Maddocks points out, this is not quite enough to say that $I$ has a constrained minimum at $y$. Remarkably, essentially the same conditions as Maddocks obtained for positive definiteness do in fact imply that $I$ has a strict local minimum at $y$ subject to the constraint $J=J(y)$, as we shall see.

For any $h \in H$ we may say $J(y+h)-J(y)=J_{y}^{\prime}(h)+\frac{1}{2} J_{y}^{\prime \prime}(h)+\epsilon_{1}(h)\|h\|^{2}$, where $\epsilon_{1}$ goes to zero as $\|h\|$ goes to zero. If we consider an $h$ for which $J(y+h)=J(y)$, then of course $0=J_{y}^{\prime}(h)+\frac{1}{2} J_{y}^{\prime \prime}(h)+\epsilon_{1}(h)\|h\|^{2}$. Now, for 
that $h$ we have

$$
\begin{aligned}
\Delta I=I(y+h)-I(y) & =I_{y}^{\prime}(h)+\frac{1}{2} I_{y}^{\prime \prime}(h)+\epsilon_{2}\|h\|^{2} \\
& =-\lambda J_{y}^{\prime}(h)+\frac{1}{2} I_{y}^{\prime \prime}(h)+\epsilon_{2}\|h\|^{2} \\
& =\frac{1}{2}\left(I_{y}^{\prime \prime}+\lambda J_{y}^{\prime \prime}\right)(h)+\left(\lambda \epsilon_{1}+\epsilon_{2}\right)\|h\|^{2} .
\end{aligned}
$$

Since $I_{y}^{\prime \prime}+\lambda J_{y}^{\prime \prime}$ is a bilinear form, there is a linear operator $A$ defined on $H$ so that $\left(I_{y}^{\prime \prime}+\lambda J_{y}^{\prime \prime}\right)(u, v)=\langle u, A v\rangle$. Similarly there is some element of $H$, call it $\nabla J$, so that $J_{y}^{\prime}$ applied to a vector $h$ is $\langle h, \nabla J\rangle$. Let $\sigma(A)$ be the spectrum of $A$. There are three cases which often arise in practice:

Theorem 1. If $\sigma(A) \cap(-\infty, c]=\emptyset$ for some $c>0$, then $I$ has a constrained minimum at $y$.

Proof. From (1) we may write $\Delta I$ as $\langle h, A h\rangle+\left(\lambda \epsilon_{1}+\epsilon_{2}\right)\|h\|^{2}$. But $\langle h, A h\rangle \geq$ $c\|h\|^{2}$ (this is easily verified using the spectral theorem, see [5]), so for $h$ sufficiently small, $\Delta I$ is positive.

Theorem 2. Suppose that $\sigma(A) \cap(-\infty, \epsilon]$ consists of a single negative eigenvalue $\lambda_{0}$ for some $\epsilon>0$. Let $\zeta$ solve $A \zeta=\nabla J$. ( $A$ will be invertible.) $I$ has a constrained minimum at $y$ if $J_{y}^{\prime}(\zeta)=\langle\zeta, A \zeta\rangle<0$, and $I$ does not have a constrained minimum at $y$ if $J_{y}^{\prime}(\zeta)=\langle\zeta, A \zeta\rangle>0$.

The proof of Theorem 2 will proceed in a series of steps.

Step 1. Assume that $\langle\zeta, A \zeta\rangle<0$. Then $I_{y}^{\prime \prime}+\lambda J_{y}^{\prime \prime}$ is strongly positive on the kernel of $J_{y}^{\prime}$.

Proof. Take $x$ in the kernel of $J_{y}^{\prime}$. As in [4], $x$ may be written as $v+\alpha \zeta$, where $v$ is perpendicular to $\varphi_{0}$, the eigenfunction corresponding to $\lambda_{0}$. (The key to this calculation is that $\left\langle\zeta, \varphi_{0}\right\rangle \neq 0$. But if $\zeta$ is orthogonal to $\varphi_{0}$, it can be shown that $\langle\zeta, A \zeta\rangle>0$.) One can verify that $\langle x, A x\rangle=\langle v, A v\rangle-\alpha^{2}\langle\zeta, A \zeta\rangle$, so that $\langle x, A x\rangle \geq\langle v, A v\rangle$.

Let $\left\{E_{\lambda}\right\}$ be the spectral family associated with $A$, so that $A=\int_{-\infty}^{\infty} \lambda d E_{\lambda}$. By our assumption on $\sigma(A), A=\lambda_{0} E_{\lambda_{0}}+\int_{\epsilon}^{\infty} \lambda d E_{\lambda}$, where $E_{\lambda_{0}}$ is orthogonal projection onto $\varphi_{0}$. Therefore,

$$
\langle v, A v\rangle=\left\langle v, \lambda_{0} E_{\lambda_{0}}(v)\right\rangle+\int_{\epsilon}^{\infty} \lambda d\left\|E_{\lambda} v\right\|^{2} .
$$

The first term vanishes, so that

$$
\langle v, A v\rangle \geq \epsilon \int_{\epsilon}^{\infty} d\left\|E_{\lambda} v\right\|^{2} \geq \epsilon \int_{-\infty}^{\infty} d\left\|E_{\lambda} v\right\|^{2} \geq \epsilon\|v\|^{2} .
$$


Therefore, $\langle x, A x\rangle \geq \epsilon\|v\|^{2}$.

To conclude the proof that $I_{y}^{\prime \prime}+\lambda J_{y}^{\prime \prime}$ is strongly positive on the kernel of $J_{y}^{\prime}$, we need to show that $\|v\| \geq k\|x\|$ for some fixed positive constant $k$. Assume without loss of generality that $\|x\|=1$. For any fixed $x,\|v\|$ is greater than or equal to the distance from $x$ to the line $\{c \zeta: c \in \mathbf{R}\}$. Consider the projection of $x$ onto $\zeta$. Its length is $|\langle x, \zeta /\|\zeta\|\rangle|$. We may write $\zeta$ as $\beta \nabla J+\hat{\zeta}$, where $\hat{\zeta}$ is perpendicular to $\nabla J$. We cannot have $\beta$ equaling 0 , since by assumption, $\langle\zeta, A \zeta\rangle=\langle\zeta, \nabla J\rangle<0$.

Then the projection has length at most $\|x\|\|\hat{\zeta}\| /\|\zeta\|$. But $\|\hat{\zeta}\|<\|\zeta\|$ (since $\beta \neq 0$ ). Letting $\gamma$ equal $\|\hat{\zeta}\| /\|\zeta\|$, we have $\gamma<1$ and the length of the vector component of $x$ perpendicular to $\zeta$ is greater than or equal to $\sqrt{1-\gamma^{2}}$. But $\|v\|$ is greater than or equal to the length of that component, so we get our $k$ to be $\sqrt{1-\gamma^{2}}$, concluding step 1 .

Step 2. If $\langle\zeta, A \zeta\rangle<0$, then $I$ has a minimum at $y$ subject to the constraint $J=J(y)$.

Proof. Take an $h$ for which $J(y+h)=J(y)$. Now $h$ need not be in the kernel of $J_{y}^{\prime}$, but we may write $h$ as $h_{1}+\alpha \zeta$, where $h_{1}$ is in the kernel of $J_{y}^{\prime}$, by taking $\alpha$ to be $\langle h, \nabla J\rangle /\langle\zeta, \nabla J\rangle$. (Note that $\langle\zeta, \nabla J\rangle=\langle\zeta, A \zeta\rangle \neq 0$.) Substituting into equation (1),

$$
\Delta I=\frac{1}{2}\left\langle h_{1}, A h_{1}\right\rangle+\alpha\left\langle h_{1}, A \zeta\right\rangle+\frac{1}{2} \alpha^{2}\langle\zeta, A \zeta\rangle+\left(\lambda \epsilon_{1}+\epsilon_{2}\right)\|h\|^{2} .
$$

However, $\left\langle h_{1}, A \zeta\right\rangle=\left\langle h_{1}, \nabla J\right\rangle=0$, causing this term to vanish. We have $0=\Delta J=J_{y}^{\prime}(h)+\epsilon_{3}\|h\|$, where $\epsilon_{3}$ tends to 0 as $\|h\|$ tends to 0 . Thus $\alpha^{2}=\epsilon_{3}^{2}\|h\|^{2}$, and we conclude that

$$
\Delta I=\frac{1}{2}\left\langle h_{1}, A h_{1}\right\rangle+\epsilon\|h\|^{2}
$$

where $\epsilon$ tends to zero as $\|h\|$ tends to 0 . From Step $1, A$ is strongly positive on the kernel of $J_{y}^{\prime}$, so

$$
\Delta I \geq \frac{k}{2}\left\|h_{1}\right\|^{2}+\epsilon\|h\|^{2} .
$$

Since $h=h_{1}+\alpha \zeta$, with $\alpha=-\epsilon_{3}\|h\|$, it is easy to see that for $\|h\|$ sufficiently small there holds $\left\|h_{1}\right\| \geq \frac{1}{2}\|h\|$. Thus

$$
\Delta I \geq\|h\|\left(\frac{k}{8}+\epsilon\right)
$$

which must be greater than 0 for $\|h\|$ sufficiently small. Therefore $I$ has a minimum at $y$ subject to the constraint $J=J(y)$, concluding the proof of step 2 and the first half of Theorem 2 . 
Step 3. Suppose that $\langle\zeta, A \zeta\rangle>0$. Then $I$ does not have a minimum at $y$ subject to the constraint $J=J(y)$.

Proof. First, $I_{y}^{\prime \prime}+\lambda J_{y}^{\prime \prime}$ is no longer positive definite on the kernel of $J^{\prime}$. Indeed, $\eta=\varphi_{0}+c \zeta$ is in the kernel of $J_{y}^{\prime}$ if $c=-\frac{\left\langle\varphi_{0}, \nabla J\right\rangle}{\langle\zeta, \nabla J\rangle}=-\frac{\left\langle\varphi_{0}, \nabla J\right\rangle}{\langle\zeta, A \zeta\rangle}$, but one can verify that $\langle\eta, A \eta\rangle<0$.

Now consider $f(r, s)=J(y+r \eta+s \nabla J)-J(y)$, a differentiable function of $r$ and $s$. Then $\nabla f(0,0)=\left(0,\|\nabla J\|^{2}\right)$, so the zero set of $f$ is tangent to the $r$ axis at the origin. From this we conclude that there is a function $s(r)$ so that $J(y+r \eta+s(r) \nabla J)-J(y)=0$, with $\lim _{r \rightarrow 0} \frac{s(r)}{r}=0$. From equation (1), for $h=r \eta+s(r) \nabla J$ we have

$$
\Delta I=\left(I^{\prime \prime}+\lambda J^{\prime \prime}\right)(r \eta+s(r) \nabla J)+\left(\lambda \epsilon_{1}+\epsilon_{2}\right)\|r \eta+s(r) \nabla J\|^{2}
$$

so that $\Delta I=r^{2}\langle\eta, A \eta\rangle+o\left(r^{2}\right)$. Thus, for all $r$ sufficiently small $\Delta I<0$, indicating that we do not have a constrained minimum, concluding the proof of Theorem 2.

Theorem 3. If $\sigma(A) \cap(-\infty, 0)$ consists of more than one point, I does not have a constrained minimum at $y$.

Proof. Suppose that $\nu$ and $\mu$ are in $\sigma(A) \cap(-\infty, 0)$, with $\nu<\mu$. Let $E_{\lambda}$ be the spectral decomposition of $A$, so that $E_{\lambda}$ is not constant in any neighborhood of $\nu$ nor in any neighborhood containing $\mu$. Take an $\epsilon>0$ so that the two $\epsilon$ neighborhoods around $\nu$ and $\mu$ are disjoint and contained in $(-\infty, 0)$. Then $E_{\nu+\epsilon}-E_{\nu-\epsilon}$ is nonzero, i.e., is a nontrivial projection. Therefore there is some $\varphi_{0} \neq 0$ so that $\left(E_{\nu+\epsilon}-E_{\nu-\epsilon}\right) \varphi_{0}=\varphi_{0}$. I claim that $\left\langle\varphi_{0}, A \varphi_{0}\right\rangle<0$.

Indeed, $\left\langle\varphi_{0}, A \varphi_{0}\right\rangle=\left\langle\varphi_{0}, \int_{-\infty}^{\infty} \lambda d E_{\lambda}\left(\varphi_{0}\right)\right\rangle$, which is $\int_{-\infty}^{\infty} \lambda d\left\langle E_{\lambda}\left(\varphi_{0}\right), \varphi_{0}\right\rangle$, where the latter just a Stieljes integral. But beyond $\nu+\epsilon, E_{\lambda}\left(\varphi_{0}\right)=\varphi_{0}$, so we only get a negative contribution. It is certainly strictly negative, since for $\lambda<\nu-\epsilon, E_{\lambda}\left(\varphi_{0}\right)=0$.

Now find a $\varphi_{1}$ for $\mu$ in the same fashion. We need to show that $\left\langle\varphi_{0}, A \varphi_{1}\right\rangle=$ 0 . But $\left\langle\varphi_{0}, A \varphi_{1}\right\rangle=\int_{-\infty}^{\infty} \lambda d\left\langle\varphi_{0}, E_{\lambda} \varphi_{1}\right\rangle$, and it is routine to show that $\left\langle\varphi_{0}, E_{\lambda} \varphi_{1}\right\rangle=0$ for all $\lambda$.

We may take $c_{0}$ and $c_{1}$, not both zero, so that $c_{0} \varphi_{0}+c_{1} \varphi_{1}$ is perpendicular to $\nabla J$. Then $\left\langle c_{0} \varphi_{0}+c_{1} \varphi_{1}, A c_{0} \varphi_{0}+A c_{1} \varphi_{1}\right\rangle=c_{0}^{2}\left\langle\varphi_{0}, A \varphi_{0}\right\rangle+c_{1}^{2}\left\langle\varphi_{1}, A \varphi_{1}\right\rangle<0$. The proof now proceeds as in Step 3 of Theorem 2.

Note. It often occurs in practice that the spectrum of $A$ is discrete and may be written as $\lambda_{0}<\lambda_{1} \leq \lambda_{2} \leq \ldots$, with 0 not a cluster point of $\sigma(A)$. In this special case, the parts of the hypotheses of the above theorems which relate to $\sigma(A)$ are as follows. In Theorem 1 we require that $0<\lambda_{0}$, in Theorem 2 we require that $\lambda_{0}<0<\lambda_{1}$ (in addition to the hypotheses on $\zeta)$, and in Theorem 3 we require that $\lambda_{0}<\lambda_{1}<0$. 


\section{References}

[1] R. Finn, Editorial comment on Stability of a Catenoidal Liquid Bridge, by Lianmin Zhou, to appear in Pac. J. Math.

[2] I.M. Gelfand and S.V. Fomin, Calculus of Variations, Prentice-Hall, Inc., Englewood Cliffs, NJ, 1963.

[3] J.H. Maddocks, Stability and Folds, Arch. Rat. Mech. Anal., 99 (1987), 301-328.

[4] Stability of nonlinear elastic rods, Arch. Rat. Mech. Anal., 85 (1984), 311354.

[5] M. Schechter, Spectra of Partial Differential Operators, North-Holland Publishing Co., Amsterdam, 1971.

Received November 17, 1994.

Texas A \& M University

College Station, TX 77840

E-mail address: tvogel@math.tamu.edu 



\title{
PACIFIC JOURNAL OF MATHEMATICS
}

\author{
Founded in 1951 by
}

\author{
$\begin{array}{ll}\text { E. F. Beckenbach (1906-1982) } & \text { F. Wolf (1904-1989) }\end{array}$
}

\section{EDITORS}

Sun-Yung A. Chang (Managing Editor) Robert Finn University of California

Los Angeles, CA 90095-1555

pacific@math.ucla.edu

\section{F. Michael Christ}

University of California

Los Angeles, CA 90095-1555

christ@math.ucla.edu

Nicholas Ercolani

University of Arizona

Tucson, AZ 85721

ercolani@math.arizona.edu
Stanford University

Stanford, CA 94305

finn@gauss.stanford.edu

Steven Kerckhoff

Stanford University

Stanford, CA 94305

spk@gauss.stanford.edu

Martin Scharlemann

University of California

Santa Barbara, CA 93106

mgscharl@math.ucsb.edu

\section{Gang Tian}

Massachusettes Institute of Technology

Cambridge, MA 02139

tian@math.mit.edu

\section{S. Varadarajan University of California Los Angeles, CA 90095-1555 vsv@math.ucla.edu \\ Dan Voiculescu \\ University of California \\ Berkeley, CA 94720 \\ dvv@math.berkeley.edu}

\section{SUPPORTING INSTITUTIONS}

ACADEMIA SINICA, TAIPEI

CALIF. INST. OF TECHNOLOGY

CHINESE UNIV. OF HONG KONG

HONG KONG UNIV. OF SCI. \& TECH.

KEIO UNIVERSITY

MACQUARIE UNIVERSITY

MATH. SCI. RESEARCH INSTITUTE

NEW MEXICO STATE UNIV.

OREGON STATE UNIV.

PEKING UNIVERSITY

RITSUMEIKAN UNIVERSITY

STANFORD UNIVERSITY

\author{
TOKYO INSTITUTE OF TECHNOLOGY \\ UNIVERSIDAD DE LOS ANDES \\ UNIV. OF ARIZONA \\ UNIV. OF BRITISH COLUMBIA \\ UNIV. OF CALIF., BERKELEY \\ UNIV. OF CALIF., DAVIS \\ UNIV. OF CALIF., IRVINE \\ UNIV. OF CALIF., LOS ANGELES \\ UNIV. OF CALIF., RIVERSIDE \\ UNIV. OF CALIF., SAN DIEGO \\ UNIV. OF CALIF., SANTA BARBARA
}

UNIV. OF CALIF., SANTA CRUZ

UNIV. OF HAWAII

UNIV. OF MELBOURNE

UNIV. OF MONTANA

UNIV. NACIONAL AUTONOMA DE MEXICO

UNIV. OF NEVADA, RENO

UNIV. OF OREGON

UNIV. OF SOUTHERN CALIFORNIA

UNIV. OF UTAH

UNIV. OF WASHINGTON

WASHINGTON STATE UNIVERSITY

The supporting Institutions listed above contribute to the cost of publication of this Journal, but they are not owners or publishers and have no responsibility for its contents or policies.

Manuscripts must be prepared in accordance with the instructions provided on the inside back cover.

The table of contents and the abstracts of the papers in the current issue, as well as other information about the Pacific Journal of Mathematics, may be found on the Internet at http://www.math.uci.edu/pjm.html.

The Pacific Journal of Mathematics (ISSN 0030-8730) is published monthly except for July and August. Regular subscription rate: $\$ 245.00$ a year (10 issues). Special rate: $\$ 123.00$ a year to individual members of supporting institutions.

Subscriptions, back issues published within the last three years and changes of subscribers address should be sent to Pacific Journal of Mathematics, P.O. Box 4163, Berkeley, CA 94704-0163, U.S.A. Prior back issues are obtainable from Kraus Periodicals Co., Route 100, Millwood, NY 10546.

The Pacific Journal of Mathematics at the University of California, c/o Department of Mathematics, 981 Evans Hall, Berkeley, CA 94720 (ISSN 0030-8730) is published monthly except for July and August. Second-class postage paid at Berkeley, CA 94704, and additional mailing offices. POSTMASTER: send address changes to Pacific Journal of Mathematics, P.O. Box 6143, Berkeley, CA 94704-0163.

\section{PUBLISHED BY PACIFIC JOURNAL OF MATHEMATICS at University of California,} Berkeley, CA 94720, A NON-PROFIT CORPORATION

This publication was typeset using AMS-LATEX,

the American Mathematical Society's TEX macro system.

Copyright (C) 1995 by Pacific Journal of Mathematics 


\section{PACIFIC JOURNAL OF MATHEMATICS}

\section{Volume $176 \quad$ No. $2 \quad$ December 1996}

One remark on polynomials in two variables

ENRIQUe ARTAl BARTOLO and PIERRETTE CASSOU-NOGUÈS

Divergence of the normalization for real Lagrangian surfaces near complex tangents XIANGHONG GONG

Classification of the stable homotopy types of stunted lens spaces for an odd prime JESUS GONZALEZ

Plancherel formulae for non-symmetric polar homogeneous spaces

JING-SONG HUANG

A uniqueness theorem for the minimal surface equation

JENN-FANG HWANG

Differential Galois groups of confluent generalized hypergeometric equations: an approach

using Stokes multipliers

Claudine Mitschi

Oscillatory theorem and pendent liquid drops

KIMIAKI NARUKAWA and TAKASHI SUZUKI

Local and global plurisubharmonic defining functions

ALAN NOELL

Specializations and a local homeomorphism theorem for real Riemann surfaces of rings

M. J. DE LA PUENTE

Eigenvalue comparisons in graph theory

GREGORY T. QUENELL

Applications of loop groups and standard modules to Jacobians and theta functions of isospectral curves

WILLI SCHWARZ

Bridged extremal distance and maximal capacity

ROBERT E. THURMAN

Imbedding and multiplier theorems for discrete Littlewood-Paley spaces

IGOR E. VERBITSKY

On constrained extrema

THOMAS VOGEL

Heat flow of equivariant harmonic maps from $\mathbb{B}^{3}$ into $\mathbb{C P} \mathbb{P}^{2}$

YUANLONG XIN

Proof of Longuerre's theorem and its extensions by the method of polar coordinates

ZHIHONG YU

Correction to: "Special generating sets of purely inseparable extension fields of unbounded exponent"

BONIFACE IHEMOTUONYE EKE 